\title{
Discretized continuous quantum-mechanical observables that are neither continuous nor discrete
}

\author{
Thais L. Silva $\odot,{ }^{1,}{ }^{*}$ Łukasz Rudnicki, ${ }^{2,3}$ Daniel S. Tasca, ${ }^{4}$ and Stephen P. Walborn ${ }^{1,5,6}$ \\ ${ }^{1}$ Instituto de Física, Universidade Federal do Rio de Janeiro, Caixa Postal 68528, Rio de Janeiro, Rio de Janeiro 21941-972, Brazil \\ ${ }^{2}$ International Centre for Theory of Quantum Technologies (ICTQT), University of Gdańsk, 80-308 Gdańsk, Poland \\ ${ }^{3}$ Center for Theoretical Physics, Polish Academy of Sciences, Aleja Lotników 32/46, 02-668 Warsaw, Poland \\ ${ }^{4}$ Instituto de Física, Universidade Federal Fluminense, Niterói, Rio de Janeiro 24210-346, Brazil \\ ${ }^{5}$ Departamento de Física, Universidad de Concepción, 160-C Concepción, Chile \\ ${ }^{6}$ ANID Millennium Science Initiative Program, Millennium Institute for Research in Optics, \\ Universidad de Concepción, 160-C Concepción, Chile
}

(Received 23 September 2020; revised 20 November 2021; accepted 23 November 2021; published 28 January 2022)

\begin{abstract}
Most of the fundamental characteristics of quantum mechanics, such as nonlocality and contextuality, are manifest in discrete, finite-dimensional systems. However, many quantum information tasks that exploit these properties cannot be directly adapted to continuous variable systems. To access these quantum features, continuous quantum variables can be made discrete by binning together their different values, resulting in observables with a finite number, $d$, of outcomes. While direct measurement indeed confirms their manifestly discrete character, here we employ a salient feature of quantum physics known as mutual unbiasedness to show that such coarse-grained observables are in a sense neither continuous nor discrete. Depending on $d$, the observables can reproduce either the discrete or the continuous behavior, or neither. To illustrate these results, we present an example for the construction of such measurements and employ it in an optical experiment confirming the existence of four mutually unbiased measurements with $d=3$ outcomes in a continuous variable system, surpassing the number of mutually unbiased continuous variable observables.
\end{abstract}

DOI: 10.1103/PhysRevResearch.4.013060

\section{INTRODUCTION}

A multitude of quantum information protocols have been developed that exploit fundamental aspects of discrete quantum systems, such as nonlocality and contextuality. However, many systems are described by continuous or infinitedimensional variables. A continuous system is fundamentally different from a discrete one, and many quantum information tasks that have been designed for discrete systems cannot be directly adapted to continuous variable (CV) systems. To overcome this, many authors have discretized the continuous variable states and/or observables, an approach that has been quite successful in terms of translating protocols that were originally cast in the discrete regime into the continuous realm. For example, it is well known that it is difficult to employ the usual phase-space operators, such as quadratures of position and momentum, to demonstrate quantum nonlocality [1]. This has led to a number of binning schemes applied to either the measurements or the states [2-7]. Similar types of discretization have been applied to quantum contextuality [8-11], the quantum search algorithm [12], entanglement

\footnotetext{
*thaisdelimasilva@gmail.com

Published by the American Physical Society under the terms of the Creative Commons Attribution 4.0 International license. Further distribution of this work must maintain attribution to the author(s) and the published article's title, journal citation, and DOI.
}

detection [13-15], and general approaches to realize finitedimensional quantum information protocols in continuous variable systems $[16,17]$.

It may be tempting to consider these discretized continuous observables as completely analogous to discrete ones, as they can be made to reproduce many of the key features. For example, clearly any direct measurement of such observables should trivially result in discrete probability distributions describing the $d$ measurement outcomes. However, when measuring different observables in distinct phase-space directions (all encoded in discrete probabilities), it might occur that correlations between the results exhibit a behavior that is characteristic of a CV system. A priori, it is not clear whether a discrete and finite-dimensional (finite number of bins) coarse graining of CVs does fully behave in a "discretelike" manner, or rather preserves some of the features of the underlying CV system.

One of these quantum features is mutual unbiasedness, where eigenstates of one measurement give equally likely outcomes for a second measurement, which displays a key distinction between discrete and continuous systems. Namely, in the continuous case, there can be at most three mutually unbiased observables with the same overlap between eigenstates of different observables [18], while for discrete systems there can be up to $d+1$ for particular values of the dimension $d$ (when $d$ is the power of a prime number) [19-22].

The distinction between the continuous and discrete cases suggests that mutual unbiasedness can be used as a benchmark for the continuous or discrete nature of coarse-grained 
observables of CV systems. In this paper we pursue this approach and discover a surprising result: In general, the observables are neither continuous nor discrete. That is, depending on the number of possible measurement outcomes, they can reproduce the discrete behavior or the continuous behavior, or give definite results that correspond to neither.

Let us briefly summarize the results we present below. We consider periodic discretization with $d$ bins, and we show that the maximum number of mutually unbiased observables is $R_{\max }=d+1$ only for prime $d$. This is also an exclusive instance which fully reproduces the discrete pattern, as in all other cases (including power-prime dimensions) the situation is more complex. However, the rule which applies in general tells us that for even dimension $d$, we get $R_{\max }=3$, imitating the $\mathrm{CV}$ case. Thus, surprisingly, we find that the investigated observables are neither continuous nor discrete, in what concerns mutual unbiasedness, at the same time showing a certain level of compatibility with both domains. An interesting aspect of our coarse graining approach is that it allows for the derivation of explicit results for any dimension parameter $d$, not just for prime power numbers, a feature yet to be achieved for discrete variable systems. We provide an accessible proof of these theoretical results, as well as an experimental demonstration of four mutually unbiased observables in a continuous system for the first time. On the one hand, our results show that discretization of continuous variables, though it may reproduce many key features of discrete systems, can also lead to unique properties that are neither continuous nor discrete. On the other hand, given an $R_{\max }$ we can find a $d$ which allows the construction of $R_{\max }$ mutually unbiased observables. Thus our work shows that it is possible to identify an arbitrary number of mutually unbiased observables in a continuous quantum system. This can have important applications in quantum tomography and randomness generation, as well as other applications.

\section{MUTUAL UNBIASEDNESS}

The original treatment $[23,24]$ of mutual unbiasedness concerns mutually unbiased bases (MUBs), namely, sets of rank 1 projectors such that the overlaps between all projectors from different sets (overlaps of elements from different bases) are equal-implying that full information according to a given basis means no information with respect to all other bases [25]. As already mentioned, discrete and finite $d$-dimensional quantum systems admit at most $d+1$ MUBs [19-22], while there are at most three MUBs for a CV system [18]. Indeed, most quantum mechanics textbooks highlight the fact that mutual unbiasedness between position $(\hat{x})$ and momentum $(\hat{p})$ operators can be demonstrated by the eigenstate projections $|\langle x \mid p\rangle|=1 / \sqrt{2 \pi}$ (we set $\hbar=1$ throughout). What is somewhat less well known is the fact that any two nonparallel phase-space operators $\hat{q}_{j}=\cos \theta_{j} \hat{x}+\sin \theta_{j} \hat{p}$ and $\hat{q}_{k}$ (defined analogously) are mutually unbiased [see Fig. 1(c)], i.e.,

$$
\left|\left\langle q_{j} \mid q_{k}\right\rangle\right|=\left(2 \pi\left|\sin \theta_{j k}\right|\right)^{-1 / 2},
$$

where $\theta_{j k} \equiv \theta_{j}-\theta_{k} \neq\{0, \pi\}$ is the angle between them [26], as illustrated in Fig. 1. Although Eq. (1) shows that there is a continuum of pairwise MUBs for a CV system, the proba-
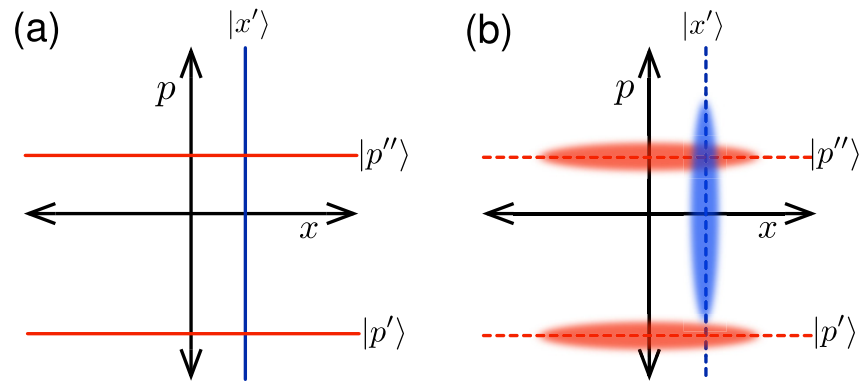

(c)

(d)
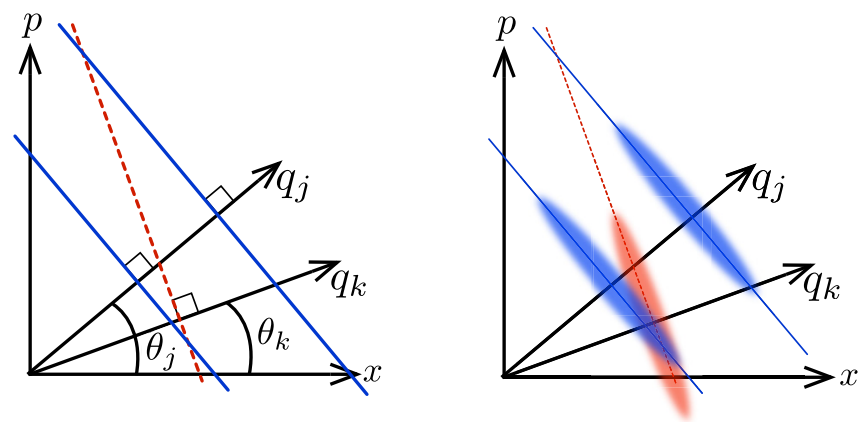

FIG. 1. Pictorial illustration of mutual unbiasedness in phase space. (a) Complementary position and momentum eigenstates are distributions denoted by the vertical and horizontal lines, respectively. The overlap between any position eigenstate and any momentum eigenstate is the same, represented by the intersection of the distributions. (b) The nonphysical eigenstates maintaining complementarity are approximated by physical states that are given by positive distributions centered around the eigenstates, with finite widths in both phase-space directions. The overlap between physical states is represented graphically by the overlap of the distributions. The physical states are no longer mutually unbiased. (c) Eigenstates of rotated observables $\hat{q}_{j}$ and $\hat{q}_{k}$, illustrating that any two nonparallel observables are mutually unbiased, as per Eq. (1). (d) Physical states corresponding to the eigenstates in (c) also lose their mutual unbiasedness.

bility of transition between states of different bases depends on the relative angle $\theta_{j k}$ between the corresponding observables. Consequently, the number of simultaneous MUBs with the same "amount" of pairwise unbiasedness is limited to three [18]. The MUBs stemming from three phase-space operators can be achieved by selecting the same relative angle $\theta_{j k}=2 \pi / 3$, such that all pairs of operators satisfy Eq. (1) with the same right-hand side [18]. Physical constraints on the eigenstates of $\hat{q}_{j}$ and $\hat{q}_{k}$ also apply, destroying the mutual unbiasedness of the corresponding physical states. In real-world experiments, they are approximated by states that are localized around some mean value (see Fig. 1). In addition, measurements in any quantum system suffer from some amount of coarse graining, which follows from the fact that every device has finite resolution. This can lead to practical consequences, such as overestimation of entanglement and/or security of quantum key distribution [27-29]. Thus mutual unbiasedness in the continuous variable regime occurs only for unphysical states, or requires consideration of measurements other than rank 1 projectors. 
There is no unique way for extending the notion of MUBs to projective measurements of rank higher than 1 and to positive operator-valued measures (POVMs) in general. In finite dimension, one alternative is offered by a a family of POVMs [30] labeled by the so-called efficiency parameter. However, these POVMs are only projective for maximal efficiency, when they boil down to rank 1 MUBs. On the other hand, every coarse graining of CV systems can be described by projective measurements (with rank $\geqslant 1$ ).

Being aware of such a discrepancy, we direct ourselves towards an experimentally oriented view on mutual unbiasedness. In particular, we resort to an operational definition [31,32], which says that a number $R$ of sets (labeled by $j$ ) of $d$ projective measurement operators, $\left\{\hat{\Omega}_{j}^{(u)}\right\}_{u=0, \ldots, d-1}$, are mutually unbiased if for all $u_{0}, u, v=0, \ldots, d-1$

$$
p_{j}^{(u)}=\delta_{u, u_{0}} \Longrightarrow p_{j^{\prime}}^{(v)}=d^{-1}, \quad j \neq j^{\prime} .
$$

By $p_{j}^{(u)}$ we denote the probability associated with a direct measurement of $\hat{\Omega}_{j}^{(u)}$. The aim of this definition is to convey the most important property of the original MUBs, namely, that localization with respect to one set implies even spreading from the perspective of all other sets. We note in passing that this definition has recently been utilized for deviceindependent applications [33].

\section{Mutual unbiasedness through periodic coarse graining}

Observables of continuous quantum variables can be discretized by a "binning" procedure. One possibility is to divide the Hilbert space into pieces of finite size (e.g., intervals). To cover the whole range of values, one needs infinitely many intervals of such type. Another option stems from dividing the space into a finite number of discrete parts. It has been shown that one path to theoretical and practical mutual unbiasedness in continuous variable systems as defined in Eq. (2) is through periodic coarse-grained (PCG) observables. With this approach, physical mutually unbiased measurement pairs [31] and mutually unbiased measurement triples [32] have been demonstrated theoretically and experimentally. In this formulation, there naturally appears a "dimensionality" parameter $d$, given by the number of possible measurement outcomes. We note also that mutually unbiased PCG measurement pairs can reproduce entropic uncertainty relations that are analogous to discrete variables [34].

Following Refs. [31,32], let us consider $R$ phase-space operators $\hat{q}_{j}$ as in Eq. (1), related to each other via phasespace rotations, each characterized by an angle $\theta_{j}$, for $j=$ $0, \ldots, R-1$, as illustrated in Figs. 2(a) and 2(b). We can then define $d$ coarse-grained projective measurement operators $(u=0, \ldots, d-1)$ :

$$
\hat{\Omega}_{j}^{(u)}=\int d q_{j} M_{j, u}\left(q_{j}-q_{j}^{\mathrm{cen}} ; T_{j}\right)\left|q_{j}\right\rangle\left\langle q_{j}\right|,
$$

where the infinite set of rank 1 projectors $\left|q_{j}\right\rangle\left\langle q_{j}\right|$ are grouped according to the $d$ non-negative "bin functions" $M_{j, u}$, such that $\sum_{u=0}^{d-1} M_{j, u}=1$. The displacement parameter $q_{j}^{\text {cen }}$ is included to allow for freedom to define the origin, and the parameter $T_{j}$ is the period of the bin function $M_{j, u}$. As in Refs. [31,32], we choose the bin functions to be periodic

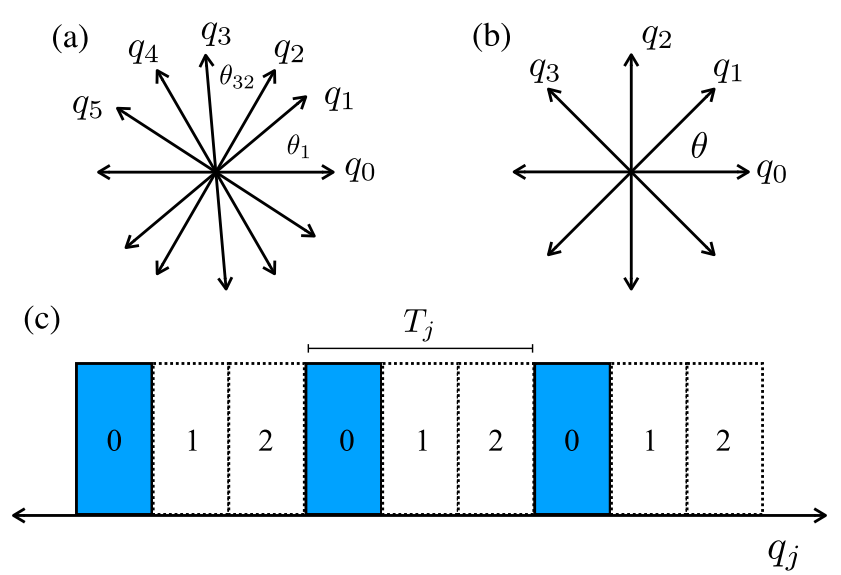

FIG. 2. Examples of phase-space variables for (a) $R=6$ and (b) the $R=4$ implemented experimentally. Here, $q_{0}$ is taken to be the position variable $x$. (c) The coarse-graining bin functions (experimentally implemented as amplitude masks) $M_{j, 0}$ for $d=3$, represented by the shaded regions.

square waves

$$
M_{j, u}(z ; T)= \begin{cases}1, & u s_{j} \leqslant z\left(\bmod T_{j}\right) \leqslant(u+1) s_{j} \\ 0, & \text { otherwise }\end{cases}
$$

specified by the period $T_{j}$ and bin width $s_{j}=T_{j} / d$, so that $d$ can indeed be considered as a dimensionality parameter. In Fig. 2(c) we illustrate the periodic bin function for the particular case of $d=3$. The outcome probabilities produced by the set of projectors (3) then define the PCG of the probability distribution associated with the phase-space variable $q_{j}$. Since we work with dimensionless variables, the parameters $T_{j}$ and $s_{j}$ are also dimensionless. We emphasize that this discretization procedure neither assumes any particular form for the $\mathrm{CV}$ system states nor is limited to a subspace, neglecting part of the entire Hilbert space [31].

Without loss of generality, we assume that $\theta_{0}=0$ and order the variables so that $\theta_{j}>\theta_{k}$ whenever $j>k$. The condition for mutual unbiasedness of these PCG operators was obtained in Ref. [32] as a linear relation between the dimensionality parameter and the product of periodicities of a pair of PCG operators:

$$
T_{j} T_{k} m_{j k}=2 \pi d\left|\sin \theta_{j k}\right|,
$$

where $\theta_{j k} \equiv \theta_{j}-\theta_{k}$ as before, and $m_{j k}$ is a positive integer. The condition (5) is supplemented by the requirement that all $m_{j k}$ are coprime with $d$, i.e.,

$$
\frac{m_{j k} n}{d} \notin \mathbb{N}, \quad \forall n=1, \ldots, d-1 .
$$

\section{RESULTS}

\section{A. Maximal number of PCG mutually unbiased measurements}

The main technical aim of this paper is to study the dependence between the maximum allowed value of $R$, such that all PCG measurements are mutually unbiased according to the operational definition (2), and the dimensionality $d$. We remind the reader that for discrete systems (instead of PCG) we know that $R \leqslant d+1$ (and the bound is tight for all $d$ that 
TABLE I. Different types of behavior for maximal number $R$ of PCG MUMs.

\begin{tabular}{ll}
\hline \hline Dimension $d$ & Pattern for maximal $R$ \\
\hline General & Subdiscrete \\
Even & Continuous \\
Odd, prime & Discrete \\
Odd, prime powers & Different \\
\hline \hline
\end{tabular}

are a power of a prime number), while for continuous systems we have $R \leqslant 3$. Therefore the way in which dimensionality bounds the number of mutually unbiased measurements (MUMs) is a signature of discrete- or continuous-type behavior. Below, we prove that for PCG,

$$
\begin{array}{ll}
R \leqslant p+1, & \text { general } d \\
R \leqslant d+1, & \text { prime } d \\
R \leqslant 3, & \text { even } d,
\end{array}
$$

where $p$ is the smallest prime factor of $d$. For composite (nonprime) dimensions, the fact that $p<d$ shows that the case of prime dimension is maximal, similarly to the discrete scenario. On the other hand, when $d$ is a prime power, i.e., $d=p^{k}$, here we still have $R \leqslant p+1$, so that the pattern known from discrete systems [19-22] is not reproduced. All distinct cases described above are collected in Table I.

Proof. Let us assume that $\sin \theta_{j} \geqslant 0$, which defines variables $q_{j}$ in the upper semiplane of the phase space. This is not a restriction, as variables in the lower half plane can be taken to the upper half plane by a reflection through the origin: $q_{j} \rightarrow-q_{j}$. Using the assumption that $\theta_{0}=0$, the $R-1$ distinct conditions stemming from Eq. (5) with $k=0$ can be rewritten in the form

$$
T_{j}=\frac{2 \pi d \sin \theta_{j}}{m_{j 0} T_{0}} .
$$

These are solutions for all periods except $T_{0}$. Plugging the above equations into (5) with $j>k>0$, we have

$$
T_{j} T_{k} m_{j k}=2 \pi d\left|\sin \theta_{j k}\right|=\frac{(2 \pi d)^{2} \sin \theta_{j} \sin \theta_{k} m_{j k}}{T_{0}^{2} m_{j 0} m_{k 0}} .
$$

This can be recast as

$$
\frac{m_{j k}}{m_{j 0} m_{k 0}}=\frac{T_{0}^{2}}{2 \pi d}\left(\cot \theta_{k}-\cot \theta_{j}\right) .
$$

There is no absolute value anymore since $\cot \theta_{k}>\cot \theta_{j}$ due to the monotonic behavior of the cotangent and the assumed ordering and range of the angles. The condition equation (10) for $k=1$ and $j \geqslant 2$ further gives

$$
\cot \theta_{j}=\cot \theta_{1}-\frac{2 \pi d}{T_{0}^{2}} \frac{m_{j 1}}{m_{j 0} m_{10}} .
$$

These are the solutions for all the angles, except the first nontrivial one. They can now be substituted into the remaining relations for $j>k>1$, producing the following set of constraints involving only natural numbers:

$$
m_{j 1} m_{k 0}-m_{j 0} m_{k 1}=m_{j k} m_{10} .
$$

Let us write $d=p p^{\prime}$, where $p$ is the smallest nontrivial prime factor of $d$, so that $p=d$ and $p^{\prime}=1$ if $d$ is prime. Observe that condition (6) implies that all $m_{j k}$ are coprime with $p$, as can be concluded by taking the particular instance $n=p^{\prime}$. This observation has two consequences. First, all $m_{j k}$ possess a modular multiplicative inverse $\tilde{m}_{j k}$ (with respect to $p$ ) which additionally is an integer. Formally, we say that $m_{j k} \tilde{m}_{j k} \equiv 1(\bmod p)$. Second, the product $m_{j k} m_{10}$ is also coprime with $p$, a fact that is mathematically expressed as $m_{j k} m_{10} \not \equiv 0(\bmod p)$. Therefore, due to $(12)$, we obtain the constraint

$$
m_{j 1} m_{k 0} \not \equiv m_{j 0} m_{k 1}(\bmod p), \quad \forall j>k>1 .
$$

Let us introduce auxiliary parameters $\chi_{k}=m_{k 0} \tilde{m}_{k 1}$, for $k=2, \ldots, R-1$. After "modulo $p$ " multiplication by the integer $\tilde{m}_{k 1} \tilde{m}_{j 1}$, Eq. (13) simplifies to the form

$$
\forall_{j>k>1} \chi_{k} \not \equiv \chi_{j}(\bmod p) \text {. }
$$

To satisfy the above condition, each $\chi_{k}$ must belong to a different congruence class modulo $p$. Therefore, at most we can have as many different values of $\chi_{k}$ as are given by the number of congruence classes, which is equal to $p-1$ since $p$ is a prime number. The final result, i.e., $R \leqslant p+1$ follows, since apart from $p$ distinct values of $\chi_{k}$ we need to include the phase-space variables $q_{0}$ and $q_{1}$. If $d$ is prime, we remember that we have $p=d$, giving $R \leqslant d+1$.

In the case of even $d$, the above result immediately implies that there are at most $R=3$ mutually unbiased PCG observables because $p=2$ for all even dimensions.

\section{B. Symmetric configuration}

There is quite a bit of freedom in the constraint (10) concerning the period $T_{0}$, as well as the angles $\theta_{j}$. Elaborating on the solution (11), with some specification, we can, however, construct a useful, symmetric recipe for mutually unbiased measurements. Let us choose angles that are distributed at integer multiples of a fixed angle $\theta$, such that $\theta \leqslant \pi / R$ and $\theta_{j}=j \theta$. Moreover, we will choose $T_{0}=\sqrt{\pi d \tan \theta}$, so that Eq. (11) divided by $\cot \theta$ assumes a simplified form

$$
\frac{\cot j \theta}{\cot \theta}=1-2 \frac{m_{j 1}}{m_{j 0} m_{10}} \text {. }
$$

The right-hand side of this condition is, by construction, a rational number. Since

$$
\frac{\cot j \theta}{\cot \theta}=\frac{\sum_{l \text { even }}(-1)^{\frac{l}{2}}\left(\begin{array}{l}
j \\
l
\end{array}\right) \tan ^{l} \theta}{\sum_{l \text { odd }}(-1)^{\frac{l-1}{2}}\left(\begin{array}{l}
j \\
l
\end{array}\right) \tan ^{l-1} \theta},
$$

we can see that the left-hand side is rational as well, whenever

$$
\tan \theta=\sqrt{Q}
$$

with $Q$ being a non-negative rational number. This is because both numerator and denominator in Eq. (16) only contain even powers of $\tan \theta$ which are equal to powers of $Q$. This construction does further allow us to adjust all $m_{j 0}$ and $m_{j 1}$.

\section{Experiment}

To confirm and explore our results, we performed an experiment using the transverse spatial degrees of freedom of 

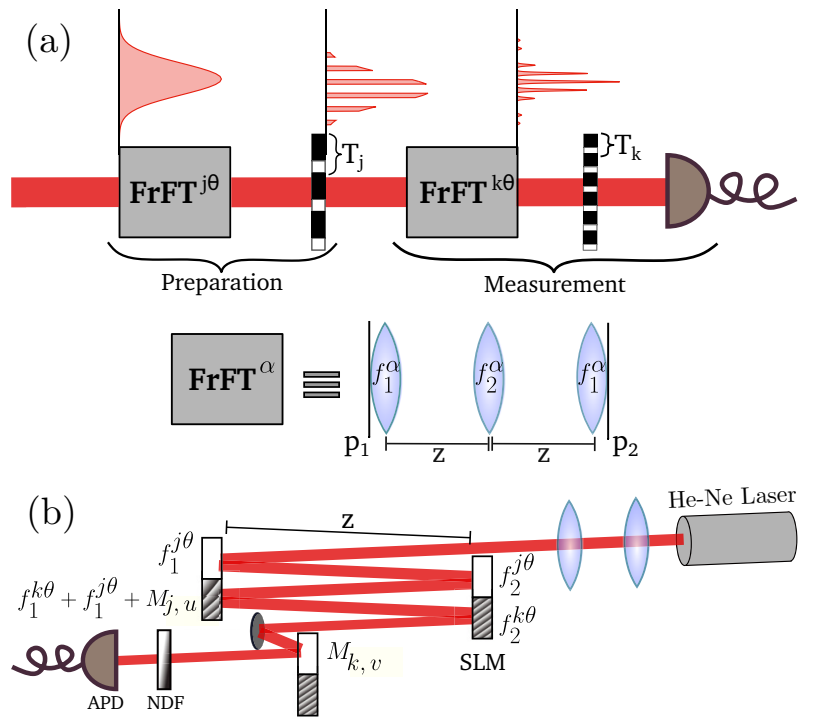

FIG. 3. (a) Schematic of the experiment. Fractional Fourier Transforms (FrFTs) and periodic amplitude masks $M$ are used to prepare and measure the transverse spatial profile of the laser beam. All output light is incident on the full-field single-photon detector. (b) Experimental setup using spatial light modulators (SLMs) for preparation and measurement. The output of a 632.8-nm He-Ne laser is enlarged and collimated using two lenses. The FrFTs are performed modulating the phase according to the quadratic phase of the lenses with the right focal distances. The use of SLMs allows us to synthesize any order FrFT, which would be challenging with actual lenses. The amplitude masks are also implemented using phase-only modulators applying diffraction gratings and collecting only the first diffraction order. Finally, the beam is attenuated with a neutral density filter (NDF) and detected by a single-photon detector [avalanche photodiode (APD)].

single photons produced by an attenuated laser source and detected by a single-photon detector. The transverse spatial degrees of freedom of a paraxial optical field are analogous to position and momentum variables in quantum mechanics [35]. Here, the near-field and far-field variables with respect to a transverse reference plane play the role of position and momentum, respectively. One can perform arbitrary rotations in the phase space formed by these variables using fractional Fourier transforms (FrFTs) [36]. In this way, we can transform the position to a variable $q_{j}$ and then an arbitrary $q_{k}$ by a sequence of two FrFTs parametrized by the angles $\theta_{j}$ and $\theta_{k j}=\theta_{k}-\theta_{j}$, respectively [37]. The projective measurements (rank $>1$ ) were realized using amplitude masks to select only the appropriate values of $q_{j}$ for each projection, following the definition of the bin functions given in Eq. (4) and illustrated in Fig. 2.

Optical FrFTs and amplitude masks were used to both prepare eigenstates with respect to one PCG measurement and perform different PCG measurements on them, as shown schematically in Fig. 3. Both the FrFTs and the amplitude masks were implemented using spatial light modulators (SLMs), as described in Appendix A. In the preparation stage, an FrFT of order $\theta_{j}$ was implemented on the transverse profile, followed by the application of an amplitude mask $M_{j, u}$ of period $T_{j}$. This corresponded to the preparation of the initial
TABLE II. Entropy value for different preparation and measurement directions in the case $R=4$ and $d=3$ with periodicities satisfying all the MU conditions (5).

\begin{tabular}{lllll}
\hline \hline \multirow{2}{*}{ Preparation } & \multicolumn{4}{c}{ Measurement } \\
\cline { 2 - 5 } & \multicolumn{1}{c}{0} & \multicolumn{1}{c}{1} & \multicolumn{1}{c}{2} & \multicolumn{1}{c}{3} \\
\hline 0 & $0.161(3)$ & $1.5846(2)$ & $1.579(1)$ & $1.5847(2)$ \\
1 & $1.5841(8)$ & $0.143(3)$ & $1.583(3)$ & $1.584(4)$ \\
2 & $1.5846(1)$ & $1.5848(1)$ & $0.140(5)$ & $1.5838(1)$ \\
3 & $1.5844(2)$ & $1.5847(1)$ & $1.5844(5)$ & $0.162(3)$ \\
\hline \hline
\end{tabular}

state $u$, in basis $j$. The measurement stage consisted of an FrFT of order $\theta_{k}$ and an amplitude mask $M_{k, v}$, projecting onto outcome $v$ in basis $k$. The full field of the resulting output beam was detected with a single-photon detector, as depicted in Fig. 3(a). In principle, the use of this FrFT scheme with SLMs allows us to perform any combination of preparation and measurement in a black-box-like manner, giving as inputs the dimension $d$, the preparation index $u \in\{0, \ldots, d-1\}$, the phase-space directions $\theta_{j}$ and $\theta_{k}$, and the respective mask periods $T_{j}$ and $T_{k}$, and obtaining as output the probability of each measurement outcome $v \in\{0, \ldots, d-1\}$.

We tested the case of $R=4$ MUMs in the symmetric configuration with dimension parameter $d=3$ and $\theta=\pi / 4$, so that $\tan \theta=1$ fulfills relation (17), for all 16 combinations of preparation and measurement. We chose the period of the zeroth mask to be $T_{0}^{\prime}=93$ pixels so that with the choice $m_{10}=m_{30}=1$ we have $T_{1}^{\prime}=T_{3}^{\prime}=132$ pixels and for $m_{20}=2$ we have $T_{2}^{\prime}=93$ pixels. These periods are the closest values in integer number of pixels to those that satisfy all conditions (10) for these $m_{j k}$ (see Appendix A).

We tested MUM conditions between preparations $j=$ $0,1,2,3$ and measurements $k=0,1,2,3$. For each measurement $k$, the detection mask was scanned in all three positions $(v=0,1,2)$, and the number of photocounts $C_{k}^{(v)}$ was registered. We then calculated the detection probabilities $p_{k}^{(v)}=$ $C_{k}^{(v)} / \sum_{v} C_{k}^{(v)}$. This was repeated for each pair of preparation and measurement observables $j$ and $k$.

To evaluate the mutual unbiasedness (MU) of the measurement results, we calculated the Shannon entropy $E=$ $-\sum_{v} p_{k}^{(v)} \log _{2} p_{k}^{(v)}$ of the probability distributions (see Appendix B). The results are shown in Table II. When the preparation basis $j$ is different from the measurement basis $k$, we obtain entropies that are close to the maximum $\log _{2} 3 \approx 1.5849$, indicating that the probability distributions are nearly uniform. To demonstrate that this is indeed due to mutual unbiasedness and not simply a result of some source of homogeneous noise, we tested the setup by realizing preparation and measurement in the same phase-space direction. In this case, we expect a deterministic probability distribution given by $p_{k}^{(v)}=\delta_{v, u}$, where $u$ is the preparation state. In all cases we observed a single probability $p_{k}^{(v=u)}>0.983(5)$ and the other two probabilities $p_{k}^{(v \neq u)}<0.0131(4)$. With a deterministic probability distribution, one expects a null value for the entropy. However, the overall $2 \%$ background noise is enough to result in the non-null entropy values shown in the diagonal of Table II. Still, this is sufficient to verify that the setup is functioning properly. Our results thus confirm 

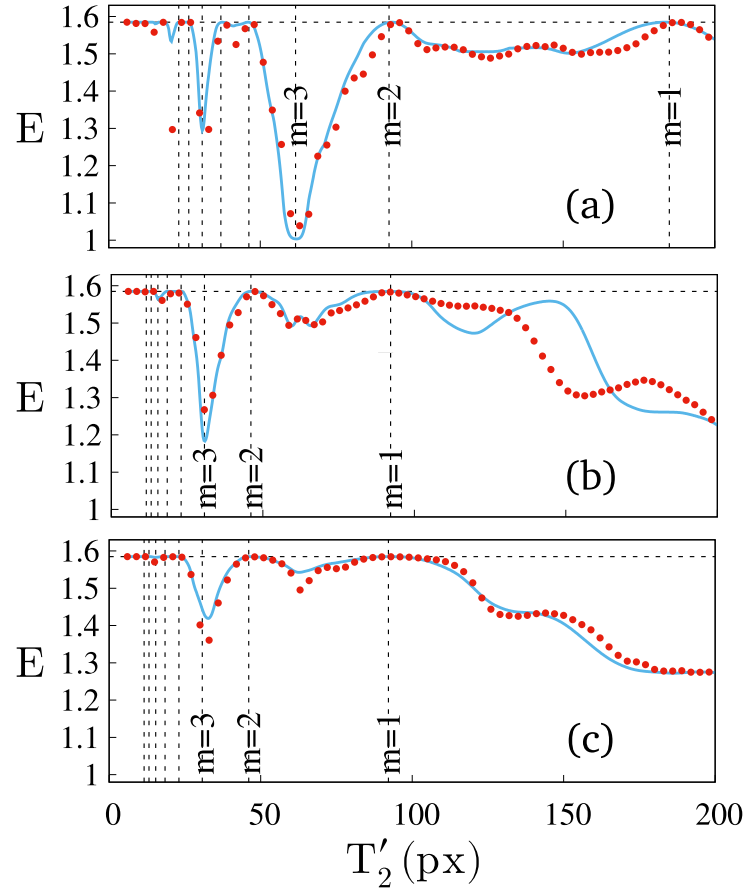

FIG. 4. Experimental results for the entropy for $R=4$ PCG measurements with a dimension parameter $d=3$, using angle $\theta=\pi / 4$. Entropy is plotted as a function of the physical period $T_{2}^{\prime}$ in units of pixels ( $\mathrm{px})$ of the measurement mask, with preparation fixed at position " 0 " and period that satisfies the mutual unbiasedness condition (8) for $m_{j 0}=1$. The measurement direction is fixed at $k=2$, and the entropy is determined for preparation directions (a) $j=0$, (b) $j=1$, and (c) $j=3$. The blue curves correspond to theoretical predictions for the experimental initial state. The red dots represent the experimental data; the error bars are smaller than the dots. The vertical dashed lines indicate the special values of the period satisfying (5) for different values of $m_{j 2}$.

mutual unbiasedness for $R=d+1=4$ PCG measurements with properly chosen periods.

To further explore mutual unbiasedness in four phase-space directions and validate our results, we tested the mutual unbiasedness conditions between preparations $j=0,1,3$ and measurement $k=2$ as a function of the period $T_{2}^{\prime}$ used in the measurement stage, obtaining the experimental results shown in Fig. 4. The theoretical prediction (solid curves in Fig. 4) is the result of integrating the theoretical probability density (such as the ones shown in Fig. 5) over the appropriate bin regions. We can see that at several places the entropy reaches its maximum value of $\log _{2} 3 \approx 1.5849$, which indicates that the probability distribution is uniform, corresponding to mutual unbiasedness. Vertical lines show values at which the period $T_{2}^{\prime}$ corresponds to allowable $m_{j 2}$ values. In all plots, we can observe that the entropy decreases greatly when $m_{j 2}=$ 3 , which is not allowed by Eq. (6) when $d=3$. Furthermore, the MUM condition is reached whenever the period $T_{2}^{\prime}$ equals 93 pixels, as expected from the derived requirements. Moreover, it corresponds to $m_{21}=m_{32}=1$ and $m_{20}=2$, as predicted by our theoretical results and previously confirmed experimentally in Table II. Similar results were obtained for all combinations of preparation and measurement. The depar-
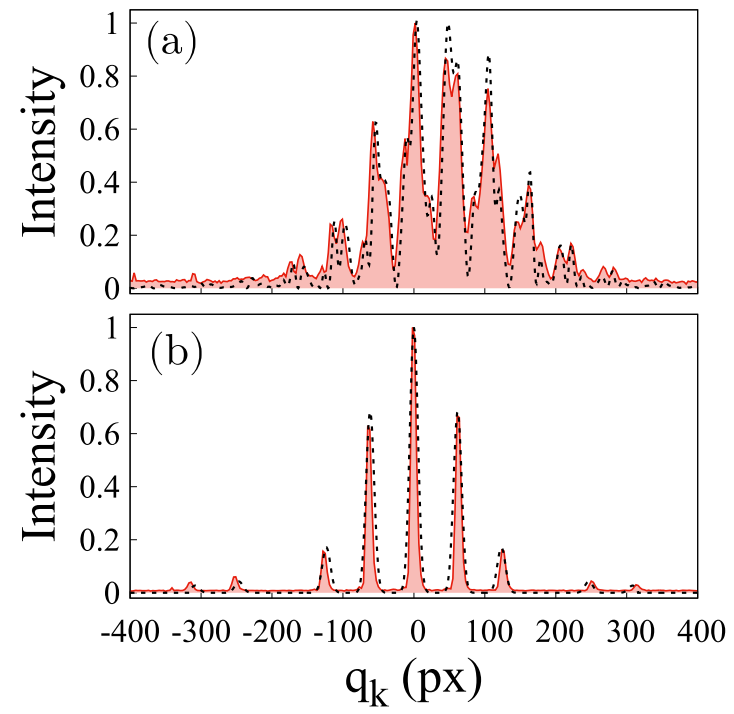

FIG. 5. Experimental intensity profiles (red solid curves) at the output SLM obtained by scanning the SLM with a narrow slit aperture while measuring the number of photocounts. The intensity profile is proportional to the probability density of $q_{k}$. The localized state prepared in direction $j=0$ is represented in directions (a) $k=1$ and (b) $k=2$. The experimental result is compared with the theoretical curves obtained by numerically propagating the initial state (dashed black curves), i.e., by applying the periodic masks and FrFTs numerically onto a Gaussian wave function representing the initial profile of the optical field.

ture of the experimental points from the theoretical curve for larger periods (fewer bins covered by the SLM screen) can be intuitively understood by considering that when the period is small, unaccounted propagation errors such as diffraction affect a large number of the bins more or less equally, so that the errors are homogeneous and do not significantly affect the output probability distribution, which is already close to uniform. However, as the period increases, the regions significantly affected by the experimental errors can become concentrated in only a few bins, which begins to modify the final probability distribution more significantly.

Let us briefly discuss experimental errors. In Fig. 4 we observe a limiting factor of our experimental implementation, and of the PCG construction in general. That is, periods $T_{j}$ satisfying condition (8) are inversely proportional to $m_{j 0}$ and thus become closer together as $m_{j 0}$ increases (small $T$ ), eventually reaching the limits imposed by the spatial resolution of our optical system. In addition to causing experimental errors, this loss of resolution sets a limit for accessible values of $m_{j 0}$. In our system this occurs at around $m_{20} \sim 14$, for which $T_{2}^{\prime}\left(m_{20}=13\right)-T_{2}^{\prime}\left(m_{20}=14\right) \approx 1$ pixel.

On the other hand, when the period is large, we essentially recover the case of standard coarse graining, where only the central set of bins have nonzero probability. Thus the interesting properties that arise from the periodicity of the bin functions are lost. In this case, additional experimental errors such as the alignment of the beam become more relevant, since with misalignment we lose mutual unbiasedness. This is not as relevant when the period is small, since the field covers many bins. Our error bars take into account only the 
uncertainty resulting from Poissonian count statistics and do not consider any other source of experimental error, such as misalignment (transverse and angular displacements). To avoid these errors, it is best to work in the middle region, where the periods are neither too large nor too small. This gives a robustness to alignment errors, as well avoiding any possible resolution issues with the spatial light modulator. We expect that other physical systems will encounter similar types of constraints.

\section{DISCUSSION}

We have shown that it is possible to define a general set of $R$ mutually unbiased observables on a continuous variable system that produces a discrete set of $d$ outcomes, while at the same time displaying behavior that is neither discrete nor continuous. This conclusion is drawn by evaluating the behavior of these measurements under conditions for mutual unbiasedness. In particular, for prime $d$ we have shown that there can be $d+1$ mutually unbiased observables, which is reminiscent of the discrete behavior. However, for composite $d$, we can have at most $p+1$, where $p$ is the smallest nontrivial prime factor of $d$. Thus, for even $d$, we can define at most three mutually unbiased measurements, which is similar to the continuous case. However, when $d=p^{k}$ is the power of a prime number, we have $p+1$, which is distinct from both the discrete and continuous cases.

An interesting aspect of this scenario is that it allows us to determine the nontrivial maximum number of mutually unbiased measurements for all values of $d$, which has not yet been achievable for discrete systems. We have corroborated and explored the practicality of our theoretical result with an experiment observing mutual unbiasedness for four measurements with $d=3$ outcomes using the transverse spatial degrees of freedom of photons. This is already beyond what can be achieved in the continuous regime. This demonstration suggests that it should be interesting to explore these types of PCG measurements in the detection and utilization of spatially entangled photon pairs outside of the usual near-field-far-field scenario [38-40]. Moreover, we expect that our results will find utility in applications that exploit unbiasedness, such as randomness generation, for example.

\section{ACKNOWLEDGMENTS}

The authors thank R. V. Nery, E. C. Paul, A. Z. Khoury, P. H. Souto Ribeiro, R. L. de Matos Filho, and G. Lima for insightful discussions. T.L.S., D.S.T., and S.P.W. acknowledge partial financial support from the Brazilian agencies CNPq (Grants No. 304196/2018-5 and No. 431804/2018-4), FAPERJ (Grants No. PDR10 E-26/202.802/2016 and No. E-26/202.7890/2017), CAPES (Grant No. PROCAD2013), and INCT-IQ (Grant No. 465469/2014-0). S.P.W. acknowledges support from the Chilean Fondo Nacional de Desarrollo Científico y Tecnológico (FONDECYT; Grant No. 1200266) and ANID Millennium Science Initiative Program ICN17_012. Ł.R. acknowledges funding by the Foundation for Polish Science (IRAP project, ICTQT, Contract No. 2018/MAB/5, cofinanced by the EU within the Smart Growth Operational Programme).

\section{APPENDIX A: EXPERIMENTAL IMPLEMENTATION OF MUTUALLY UNBIASED MEASUREMENTS}

The initial state in position is fixed and is prepared as a collimated Gaussian beam with beam radius $(2.54 \pm 0.06) \mathrm{mm}$ at the plane of the first SLM. The beam was prepared and measured with rotations in phase space, performed with FrFTs, and amplitude masks. To realize the FrFTs, we use the scheme described in detail in Ref. [41], where SLMs are used to imitate three lenses by imprinting quadratic phases on the optical field (see Fig. 3). The lenses are separated by a distance $z$. If the focal distances satisfy $f_{1}^{\alpha}=z\left(1-\frac{\cot (\alpha / 2)}{2}\right)^{-1}$ and $f_{2}^{\alpha}=z(1-\sin \alpha)^{-1}$, then the transverse profile of the optical field in the plane $p_{2}$ is the FrFT of order $\alpha$ of the field in the plane $p_{1}$ up to a scaling factor that is independent of the FrFT order. The SLMs were also used to imprint amplitude masks with physical period $T_{j}^{\prime}$, which define the discrete bins in our measurements. Using the three-lens FrFT scheme introduces a scaling factor independent of the rotation angle, such that dimensionless $\left(T_{j}\right)$ and physical $\left(T_{j}^{\prime}\right)$ periods are related by $T_{j}^{\prime}=\sqrt{\frac{\lambda z}{\pi}} T_{j}$, where $z=0.29 \mathrm{~m}$ is the distance between the lenses and $\lambda=632.9 \mathrm{~nm}$ is the $\mathrm{He}-\mathrm{Ne}$ laser wavelength. Moreover, for practical reasons, the physical periods are given in units of SLM pixels. The pixel size of the Holoeye SLMs used here is $8 \mu \mathrm{m}$. We chose the period of the zeroth mask to be $T_{0}^{\prime}=93$ pixels, since this value is the closest integer number to the value of the exact solution (92.7476 pixels). Using (8), and choosing $m_{10}=m_{30}=1$, we have $T_{1}^{\prime}=T_{3}^{\prime}=131.165$ pixels, approximated by 132 pixels so the bin width is an integer number of pixels. For $m_{20}=2$ we have $T_{2}^{\prime}=92.7476 \approx 93$ pixels. These values of $m_{j k}$ satisfy all conditions (10).

To test the experimental setup, we scanned the output SLM with a narrow slit aperture so that we could measure the final transverse intensity profile of light produced by the optical transformations acting on the initial state (all but fi-
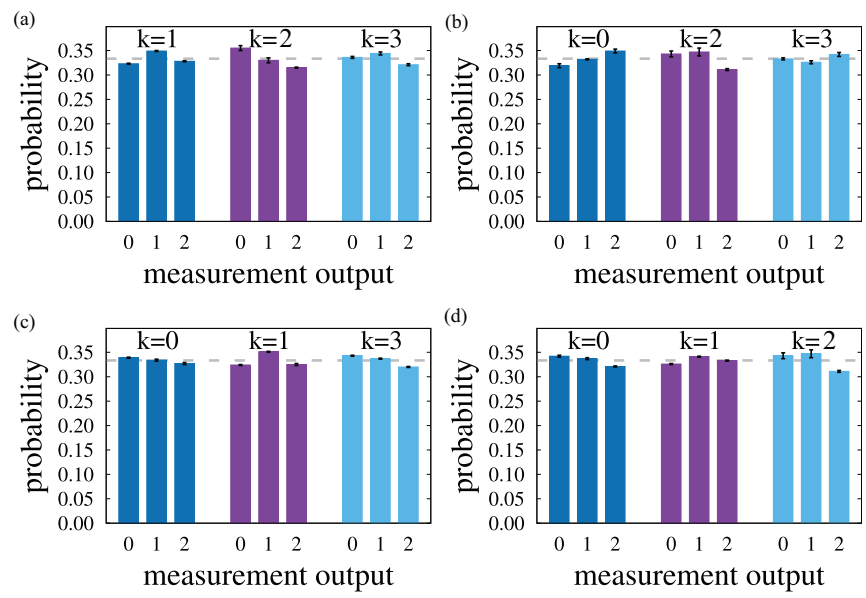

FIG. 6. Experimental outcome probabilities used to calculate the Shannon entropies shown in Table II for preparation directions (a) $j=0$, (b) $j=1$, (c) $j=2$, and (d) $j=3$. Only the cases when the measurement direction $k$ is different from that used to prepare the localized state are presented. The dashed horizontal line corresponds to $p=\frac{1}{d}=\frac{1}{3}$. 
nal amplitude mask $M_{k, v}$ ). Some results are exemplified in Fig. 5. This intensity profile should be proportional to the probability density obtained from the theoretical initial state propagated through the preparation mask and two FrFTs. In this way, Fig. 5 reveals the qualitative agreement between our theoretical description and the optical setup. The theoretical probability densities obtained as described are used to predict the results when the PCG measurements are performed.

\section{APPENDIX B: EXPERIMENTAL PROBABILITY DISTRIBUTIONS}

For the case when the four PCG measurements satisfy the pairwise MUM condition, the values of the Shannon entropy shown in Table II are close to the maximum of $\log _{2}(d)$ suggesting that in that case we obtained flat probability distributions or unbiasedness. Figure 6 shows the probability distributions obtained in our experiment. For all combinations $j, k$ of preparation and measurement directions we observe a nearly flat distribution. It can be confirmed further by calculating the Kullback-Leibler divergence from the ideal uniform
TABLE III. Kullback-Leibler divergence $\left(10^{-3}\right)$ from the uniform distribution of the probability distributions of Fig. 6.

\begin{tabular}{lllll}
\hline \hline \multirow{2}{*}{ Preparation } & \multicolumn{4}{c}{ Measurement } \\
\cline { 2 - 5 } 0 & \multicolumn{1}{c}{0} & \multicolumn{1}{c}{1} & \multicolumn{1}{c}{2} & \multicolumn{1}{c}{3} \\
\hline 1 & & $0.65(2)$ & $1.3(3)$ & $0.4(2)$ \\
2 & $0.7(3)$ & & $1.1(3)$ & $0.2(1)$ \\
3 & $0.13(5)$ & $0.70(9)$ & & $0.45(6)$ \\
\hline \hline
\end{tabular}

distribution with

$$
D(P \| Q)=\sum_{v=0}^{d-1} p^{(v)} \log _{2}\left(\frac{p^{(v)}}{q^{(v)}}\right),
$$

where $p^{(v)}$ is the experimental probability of outcome $v$ and $q^{(v)}=\frac{1}{d}$ is the uniform distribution. The values of the Kullback-Leibler divergence for the unbiased PCG configuration are below 0.0013(3) as can be seen in Table III.
[1] M. Revzen, P. A. Mello, A. Mann, and L. M. Johansen, Bell's inequality violation with non-negative Wigner functions, Phys. Rev. A 71, 022103 (2005).

[2] A. Gilchrist, P. Deuar, and M. D. Reid, Contradiction of Quantum Mechanics with Local Hidden Variables for Quadrature Phase Amplitude Measurements, Phys. Rev. Lett. 80, 3169 (1998).

[3] K. Banaszek and K. Wódkiewicz, Nonlocality of the EinsteinPodolsky-Rosen state in the Wigner representation, Phys. Rev. A 58, 4345 (1998).

[4] K. Banaszek and K. Wódkiewicz, Testing Quantum Nonlocality in Phase Space, Phys. Rev. Lett. 82, 2009 (1999).

[5] J. Wenger, M. Hafezi, F. Grosshans, R. Tualle-Brouri, and P. Grangier, Maximal violation of Bell inequalities using continuous-variable measurements, Phys. Rev. A 67, 012105 (2003).

[6] D. Cavalcanti, N. Brunner, P. Skrzypczyk, A. Salles, and V. Scarani, Large violation of Bell inequalities using both particle and wave measurements, Phys. Rev. A 84, 022105 (2011).

[7] A. Ketterer, A. Keller, T. Coudreau, and P. Milman, Testing the Clauser-Horne-Shimony-Holt inequality using observables with arbitrary spectrum, Phys. Rev. A 91, 012106 (2015).

[8] S. Massar and S. Pironio, Greenberger-Horne-Zeilinger paradox for continuous variables, Phys. Rev. A 64, 062108 (2001).

[9] A. R. Plastino and A. Cabello, State-independent quantum contextuality for continuous variables, Phys. Rev. A 82, 022114 (2010).

[10] A. Asadian, C. Budroni, F. E. S. Steinhoff, P. Rabl, and O. Gühne, Contextuality in Phase Space, Phys. Rev. Lett. 114, 250403 (2015).

[11] A. Laversanne-Finot, A. Ketterer, M. R. Barros, S. P. Walborn, T. Coudreau, A. Keller, and P. Milman, General conditions for maximal violation of non-contextuality in discrete and continuous variables, J. Phys. A: Math. Theor. 50, 155304 (2017).

[12] A. Ketterer, T. Douce, A. Keller, T. Coudreau, and P. Milman, Quantum search with modular variables, arXiv:1407.1298 [quant-ph].
[13] C. Gneiting and K. Hornberger, Detecting Entanglement in Spatial Interference, Phys. Rev. Lett. 106, 210501 (2011).

[14] M. A. D. Carvalho, J. Ferraz, G. F. Borges, P.-L. de Assis, S. Pádua, and S. P. Walborn, Experimental observation of quantum correlations in modular variables, Phys. Rev. A 86, 032332 (2012).

[15] D. S. Tasca, Ł. Rudnicki, R. S. Aspden, M. J. Padgett, P. H. Souto Ribeiro, and S. P. Walborn, Testing for entanglement with periodic coarse graining, Phys. Rev. A 97, 042312 (2018).

[16] P. Vernaz-Gris, A. Ketterer, A. Keller, S. P. Walborn, T. Coudreau, and P. Milman, Continuous discretization of infinite-dimensional Hilbert spaces, Phys. Rev. A 89, 052311 (2014).

[17] A. Ketterer, A. Keller, S. P. Walborn, T. Coudreau, and P. Milman, Quantum information processing in phase space: A modular variables approach, Phys. Rev. A 94, 022325 (2016).

[18] S. Weigert and M. Wilkinson, Mutually unbiased bases for continuous variables, Phys. Rev. A 78, 020303(R) (2008).

[19] I. D. Ivonovic, Geometrical description of quantal state determination, J. Phys. A: Math. Gen. 14, 3241 (1981).

[20] W. K. Wootters and B. D. Fields, Optimal state-determination by mutually unbiased measurements, Ann. Phys. (Amsterdam) 191, 363 (1989).

[21] S. Bandyopadhyay, P. Boykin, V. Roychowdhury, and F. Vatan, A new proof of the existence of mutually unbiased bases, Algorithmica 34, 512 (2002).

[22] A. Klappenecker and M. Rötteler, Constructions of mutually unbiased bases, in Finite Fields and Applications, edited by G. Mullen, A. Poli, and H. Stichtenoth (Springer, New York, 2003), p. 137

[23] J. Schwinger, Unitary operator bases, Proc. Natl. Acad. Sci. USA 46, 570 (1960).

[24] K. Kraus, Complementary observables and uncertainty relations, Phys. Rev. D 35, 3070 (1987).

[25] T. Durt, B.-G. Englert, I. Bengtsson, and K. Życzkowski, On mutually unbiased bases, Int. J. Quantum Inf. 08, 535 (2010). 
[26] We note that in the limit $\theta_{j k} \rightarrow 0$, the limit must be taken before the absolute value to recover the normalization to the usual Dirac delta function.

[27] D. S. Tasca, Ł. Rudnicki, R. M. Gomes, F. Toscano, and S. P. Walborn, Reliable Entanglement Detection under Coarse-Grained Measurements, Phys. Rev. Lett. 110, 210502 (2013).

[28] M. R. Ray and S. J. van Enk, Missing data outside the detector range: Continuous-variable entanglement verification and quantum cryptography, Phys. Rev. A 88, 042326 (2013).

[29] M. R. Ray and S. J. van Enk, Missing data outside the detector range. II. Application to time-frequency entanglement, Phys. Rev. A 88, 062327 (2013).

[30] A. Kalev and G. Gour, Mutually unbiased measurements in finite dimensions, New J. Phys. 16, 053038 (2014).

[31] D. S. Tasca, P. Sánchez, S. P. Walborn, and Ł. Rudnicki, Mutual Unbiasedness in Coarse-Grained Continuous Variables, Phys. Rev. Lett. 120, 040403 (2018).

[32] E. C. Paul, S. P. Walborn, D. S. Tasca, and Ł. Rudnicki, Mutually unbiased coarse-grained measurements of two or more phase-space variables, Phys. Rev. A 97, 052103 (2018).

[33] A. Tavakoli, M. Farkas, D. Rosset, J.-D. Bancal, and J. Kaniewski, Mutually unbiased bases and symmetric informationally complete measurements in Bell experiments, Sci. Adv. 7, eabc3847 (2021).
[34] Ł. Rudnicki and S. P. Walborn, Entropic uncertainty relations for mutually unbiased periodic coarse-grained observables resembling their discrete counterparts, Phys. Rev. A 104, 042210 (2021).

[35] Light Transmission Optics, edited by D. Marcuse (Van Nostrand Reinhold, New York, 1982).

[36] D. S. Tasca, R. M. Gomes, F. Toscano, P. H. Souto Ribeiro, and S. P. Walborn, Continuous-variable quantum computation with spatial degrees of freedom of photons, Phys. Rev. A 83, 052325 (2011).

[37] H. M. Ozaktas, Z. Zalevsky, and M. A. Kutay, The Fractional Fourier Transform: With Applications in Optics and Signal Processing (Wiley, New York, 2001).

[38] D. S. Tasca, S. P. Walborn, P. H. Souto Ribeiro, and F. Toscano, Detection of transverse entanglement in phase space, Phys. Rev. A 78, 010304(R) (2008).

[39] D. S. Tasca, S. P. Walborn, P. H. Souto Ribeiro, F. Toscano, and P. Pellat-Finet, Propagation of transverse intensity correlations of a two-photon state, Phys. Rev. A 79, 033801 (2009).

[40] E. C. Paul, D. S. Tasca, Ł. Rudnicki, and S. P. Walborn, Detecting entanglement of continuous variables with three mutually unbiased bases, Phys. Rev. A 94, 012303 (2016).

[41] J. A. Rodrigo, T. Alieva, and M. L. Calvo, Programmable twodimensional optical fractional fourier processor, Opt. Express 17, 4976 (2009). 